\title{
Immunization in Alzheimer's disease: naïve hope or realistic clinical potential?
}

\author{
JK Foster ${ }^{1,2,3,4}$, G Verdile ${ }^{1,2,3}$, KA Bates $^{1,2,3}$ and RN Martins ${ }^{1,2,3}$ \\ ${ }^{1}$ The Sir James McCusker Alzheimer's Disease Research Unit, Hollywood Private Hospital, Hollywood, WA, Australia; ${ }^{2}$ Centre \\ of Excellence for Alzheimer's Disease Research and Care, School of Exercise, Biomedical and Health Sciences, Edith Cowan \\ University, Joondalup, WA, Australia; ${ }^{3}$ School of Psychiatry and Clinical Neurosciences, University of Western Australia, \\ Crawley, WA, Australia and ${ }^{4}$ Neurosciences Unit, Health Department of Western Australia, Mount Claremont, WA, Australia
}

\begin{abstract}
There has been considerable recent interest in vaccination of patients by immunotherapy as a potentially clinically useful methodology for combating histopathological changes in Alzheimer's disease (AD). The focus of the majority of this research has been on (1) active immunotherapy using the pre-aggregated synthetic $\beta$-amyloid (A $\beta$ ) 42 preparation AN1792 vaccine (QS-21), or (2) passive immunization using injections of already prepared polyclonal anti-A $\beta$ antibodies (intravenous immunoglobulin). These two clinical approaches to the treatment of patients with AD represent the focus of this review. We conclude here that, with certain caveats, immunization offers further potential as a technique for the treatment (and possible prevention) of AD. New studies are seeking to develop and apply safer vaccines that do not result in toxicity and neuroinflammation. Nevertheless, caution is warranted, and future clinical investigations are required to tackle key outstanding issues. These include the need to demonstrate efficacy in humans as well as animal models (especially with respect to the potentially toxic side effects of immunotherapy), and fine-tuning in safely guiding the immune response. The issue of defining necessary and sufficient criteria for determining clinical efficacy remains an additional important issue for future immunization trials. The vaccination methodology appears to offer substantial current promise for clearing both soluble and aggregated amyloid in AD. However, it remains to be determined whether this approach will help to repair already damaged neural systems in the disease, and the extent to which vaccination-driven amyloid clearance will impact beneficially on patients' neurocognitive capacity and their functional status. The outcomes of future studies will be important both clinically and scientifically: an important further test of the validity of the amyloid hypothesis of AD is to evaluate the impact of an effective anti-amyloid strategy on the functional status of patients with this disease.

Molecular Psychiatry (2009) 14, 239-251; doi:10.1038/mp.2008.115; published online 28 October 2008
\end{abstract}

Keywords: dementia; immunotherapy; $\beta$-amyloid; brain; cognition

\section{Background}

Vaccination has been established as a valuable clinical prevention method for over 200 years. Jenner developed the first effective vaccine against smallpox (the only disease to be eliminated by modern medicine) in the late eighteenth century, whereas in the nineteenth and twentieth centuries effective vaccines were developed against rabies and polio that have effectively neutralized these illnesses worldwide. Treatment with antibodies has also proven effective in autoimmune illnesses such as multiple sclerosis, indicating that a degenerative

Correspondence: Dr JK Foster, Centre of Excellence for Alzheimer's Disease Research and Care, School of Exercise, Biomedical and Health Sciences, Edith Cowan University, 100 Joondalup Drive, Joondalup, WA 6027, Australia.

E-mail: j.foster@ecu.edu.au

Received 7 March 2007; revised 6 October 2008; accepted 7 October 2008; published online 28 October 2008 disease of the central nervous system (CNS) may be tractable to vaccination. By contrast, current pharmacological treatments for Alzheimer's disease (AD) provide modest symptomatic benefit for some patients, but do little to slow disease progression. ${ }^{1}$ Consequently, there has been considerable recent interest in immunotherapy as a potentially useful clinical methodology for combating histopathological changes in AD. Within this field, there has been interest-inter alia-in monoclonal antibody therapy, cytokines, memapsin 2 antibodies, the sendai virus vector, $\beta$-amyloid (A $\beta$ ) phagocytosis through microglia, phage therapy, catalytic autoantibodies, immunomodulatory drugs, $\alpha$-synuclein antibodies, prion-mucosal therapy, DNA vaccines, 'snapshot' antibodies, immunoconjugates and chimeric proteins. However, the majority of recent research into potential vaccination therapies has focused on (1) active immunization using the pre-aggregated synthetic A $\beta 1-42$ preparation AN1792 vaccine (QS-21) or (2) 
passive immunization using injections of already prepared polyclonal anti-antibodies (for example, intravenous immunoglobulin, IVIg). These approaches represent the focus of this review, concentrating on the potential clinical efficacy of immunotherapy for sustaining neurocognitive capacity in AD patients.

The rationale underlying those AD immunization studies conducted to date has been based on the influential 'amyloid hypothesis' of AD, which hypothesizes that an imbalance in the production (and subsequent accumulation and aggregation) or clearance of $A \beta 1-42$ is the major cause of neuronal loss and dysfunction underlying dementia of the Alzheimer type. ${ }^{2}$ From a scientific perspective, the field of immunotherapy offers the potential to provide important information concerning the validity of the amyloid hypothesis of $\mathrm{AD}$. In addition, amyloidrelated ante-mortem diagnosis using clinical techniques such as PIB-PET has the potential to offer further valuable information concerning the amyloid hypothesis of AD pathophysiology. ${ }^{3}$ Moreover, there is now significant evidence that amyloid deposition initiates the hyperphosphorylation of tau. This process represents the pathophysiological mechanism underlying the formation of neurofibrillary tangles-an additional pathological hallmark found among degenerating neuronal cells in AD. ${ }^{4}$

Considerable evidence has accumulated indicating that $A \beta$ is neurotoxic, and that its accumulation initiates a cascade of events that ultimately lead to synaptic failure and neuronal death. ${ }^{5}$ In addition, it has been suggested that there is defective innate immunity to $\mathrm{A} \beta$ in $\mathrm{AD} .{ }^{6} \mathrm{~A}$ number of risk factors have been associated with increased incidence of $\mathrm{AD}$, including genetic status (for example, possession of the APOE\&4 allele), type II diabetes, cardiovascular factors (such as hypertension), sedentary lifestyle, head injury, female gender, dietary cholesterol and reductions in endogenous hormones (such as estrogen and testosterone) that regulate $\mathrm{A} \beta$ metabolism. ${ }^{7,8} \mathrm{~A}$ common feature with respect to these risk factors is that they are associated with increased levels of $A \beta$ in the brain, offering further support for the association between $A \beta$ and $A D .^{9,10}$

The amyloid hypothesis underlies many current drug development initiatives for AD. These initiatives are becoming increasingly important, as the burden of $\mathrm{AD}$ reaches an estimated 4.5 million individuals in the United States (with the cost of the disease considered to be approximately \$US100 billion annually). However, a critical test for the validity of the amyloid hypothesis is whether these anti-amyloid strategies (which remove amyloid from the CNS) improve cognition and functional status in AD patients. An anti-amyloid vaccine that is safely able to clear amyloid plaques in AD would likely represent a major development, both clinically and scientifically. Such a vaccine would not only offer potential utility as a treatment, but it would also enable us to gauge empirically whether the accumulation of $A \beta$ in the AD brain is more likely to be (1) a key pathogenic agent of the disease or, alternatively, (2) a by-product with limited causal significance. The findings of such studies alone will not necessarily be definitive in this regard. However, immunotherapy studies are likely to offer valuable information with respect to the pathophysiological mechanisms underlying AD. Indeed, if future clinical trials continue to completion, they may offer both a direct therapeutic evaluation of the amyloid hypothesis in AD patients, and a potentially effective clinical treatment for the disease.

It has been reported that immunization with the pre-aggregated synthetic A $\beta 42$ preparation AN1792 vaccine (QS-21) reduces $A \beta$ plaque burden (and associated astrogliosis and neuritic dystrophy) and preserves cognitive functioning in several different transgenic mouse models. ${ }^{11-16}$ Moreover, data have been published showing that immunization can encourage endogenous neurogenesis in AD transgenic mice. ${ }^{17}$ The behaviors measured in these studies tapped improved reference memory for a hidden platform in a water maze ${ }^{12}$ and enhanced performance on the radial arm water-maze test of working memory. ${ }^{13}$ However, there is a considerable gulf between neurocognitive capacity in humans and other animal species, not least because of the major role played by language in mediating high-level human cognitive functioning. ${ }^{18}$ Furthermore, animal models do not mimic AD with respect to amyloid clearance: vaccination of animals removes foreign (that is, human) amyloid whereas vaccination in humans targets endogenous amyloid deposits. Therefore, it is critically important to evaluate efficacy of immunotherapy on neurocognitive symptoms in human patients: this issue represents the focus of this review.

If immunotherapy were to be effective in humans, administration of a vaccine should halt or at least slow cognitive and clinical decline in human patients. The mechanisms underlying the beneficial effects of immunotherapy observed in animal models remains poorly understood. However, it has been suggested that the efficacy of immunization with AN1792 in the PDAPP mouse model supports this approach as a potential therapeutic strategy for the treatment of $\mathrm{A} \beta$ deposition in $\mathrm{AD}$ patients. Nevertheless, studies with humans to date remain somewhat equivocal, not least with respect to the findings obtained from a large multicenter phase II clinical trial conducted over the past several years. ${ }^{19}$ Of relevance here may be the observation that tissue amyloid plaque immunoreactivity (TAPIR; which correlates with stabilization of cognitive decline in human AD patients) may be somewhat limited in those animal species used in laboratory investigations (nonhuman primates, other mammals) that lack spontaneously preexisting amyloid plaques. ${ }^{20} \mathrm{We}$ therefore focus here on investigations of the impact of immunotherapy on $A \beta$ levels and neurocognitive status in $\mathrm{AD}$ patients, considering findings obtained from both 'active' and 'passive' forms of immunotherapy. (In active immunotherapy, the onus is on the 
immune system to create antibodies of its own in $\mathrm{AD}$, whereas in passive immunotherapy, a preprepared injection of antibodies is administered to AD patients).

\section{Active immunization}

In April 2000, a phase 1 clinical trial investigating a synthetic preparation of the potential therapeutic agent $A \beta 42$ (AN1792) was conducted across four sites in the United Kingdom. This was a randomized, multiple-dose, dose-escalation, double-blind trial (sponsored by Élan-Wyeth) that evaluated 80 patients with mild-moderate AD. The initial findings were promising. There were few adverse effects, a possible improvement in one of the clinical measures and demonstrated immunogenicity of the compound. The trial was then extended to phase IIa in October 2001, involving some 30 centers in the United States, Europe and United Kingdom. However, the trial was halted by the sponsors in January 2002 after four patients developed meningoencephalitis. After the trial was halted, it was determined that 18 patients in total $(6 \%)$ had developed meningoencephalitis, all of whom were in the active treatment group. Despite this outcome, there have been a number of further papers reporting the clinical, cognitive and neuropathological consequences of AN1792 treatment. These are summarized below.

Gilman et $a l .{ }^{19}$ reported an analysis of the phase IIa immunotherapy trial of AN1792 (QS-21) in patients with probable mild to moderate AD. In this trial, 300 patients diagnosed with $\mathrm{AD}$ received the active AN1792 treatment (that is, $0.5 \mathrm{ml}$ intramuscular injection of $225 \mu \mathrm{g}$ A $\beta 42$ with $50 \mu \mathrm{g}$ QS-21 adjuvant), whereas $74 \mathrm{AD}$ patients received saline placebo. The original protocol involved six injections over a 12-month period, with (1) cognitive change, measured using the Alzheimer's Disease Assessment ScaleCognitive Part (ADAS-Cog), Mini Mental State Examination (MMSE) and a Neuropsychological Test Battery (NTB), and (2) change in whole-brain volume using serial magnetic resonance imaging (MRI) designated as the two main outcome variables, along with safety and tolerability assessment. The immunogenic response to AN1792 treatment was measured by enzyme-linked immunosorbent assay in blood serum and cerebrospinal fluid (CSF), whereby responders were defined as patients who developed a serum AN1792 immunoglobulin G (IgG) titer of $\geqslant 1: 2200$ at any time point after the initial injection.

The findings of the study demonstrated that patients can respond to immunization through increasing antibody titers to $A \beta$ antigens, and provided some suggestion that vaccination might improve aspects of cognition. However, similar to the reports of an earlier investigation undertaken by Orgogozo et $a l .{ }^{21}$ the study conducted by Gilman et al. ${ }^{19}$ was terminated prematurely because of a significant adverse event: subacute aseptic meningoencephalitis (a serious sign of CNS inflammation) was observed in
$6 \%(n=18)$ of patients immunized with AN1792. It appears that this reaction may have been because of the activation of cytotoxic $\mathrm{T}$ cells and/or to autoimmune reactions. Furthermore, it has been reported that of the patients who developed meningoencephalitis in the study conducted by Gilman et al. ${ }^{19}$ several of these individuals sustained long-term cognitive and/or neurological deficits. It appears likely that the incidence of encephalitis was linked to the vaccination protocol, as encephalitis had not been observed previously in post-mortem AD tissue before this trial. Notwithstanding this issue, the study conducted by Gilman et al. ${ }^{19}$ generated some other interesting findings. Specifically, of the 300 patients with diagnosed AD that were treated with AN1792 (QS-21) in this study, it was reported that $19.7 \%$ developed the predetermined antibody response to $A \beta$ antigens. There were no significant differences between antibody responders and placebo groups on the cognitive and clinical measures that were used in this study (ADAS-Cog, Disability Assessment for Dementia (DAD), Clinical Dementia Rating scale, MMSE or Clinical Global Impression of Change, CGIC). However, antibody responders had a slower rate of decline in terms of their score on the nine-item composite NTB compared to placebo patients (although it should be noted that only a relatively small number of participants in the trial completed the full NTB). More specifically, among those patients reported by Gilman et al. ${ }^{19}$ who did manifest an immune response, individuals who produced relatively high levels of antibodies to $A \beta$ antigens achieved better results in terms of their memory capacity (based on a composite score) than did those individuals who produced lower levels of antibodies to $A \beta$ antigens. (There was, additionally, some indication of a doseresponse relationship.) This is an especially interesting finding, given the identification of memory dysfunction as an early stage feature of AD. ${ }^{22}$ Overall, higher IgG antibody titers were associated with greater improvements from baseline for the (1) overall composite neuropsychology z-score, as well as for the (2) memory, (3) immediate memory and (4) delayed memory composite neuropsychology $Z$-scores. In addition, in the small subset of subjects who received CSF examinations $(n=11$ antibody responders and $n=10$ who received placebo), CSF tau was shown to be decreased from baseline in antibody responders. Gilman et al. ${ }^{19}$ cautiously interpreted their overall findings as indicating that AN1792 (QS-21) antibody responders had a slower rate of neuronal death and cognitive decline, compared to those individuals receiving placebo.

A major surprise in the outcome of the clinical trial of AN1792 came from the subsequent neuroimaging findings, as reported by Fox et $a .^{23}$ The focus of this component of the investigation was to assess MRI-derived cerebral volume changes (as a biomarker of disease progression) in patients immunized with AN1792. It was noted in this study that rates of brain atrophy measured by serial MRI increased 
successively from first symptoms through mild cognitive impairment to diagnosed AD. However, using planimetry with rigid and fluid registration to measure brain volumes, Fox et $a .^{23}$ reported that AN1792-treated patients who were identified as antibody responders to $A \beta$ antigens manifested (1) greater brain volume decrease over the course of treatment, (2) greater ventricular enlargement as a percentage of baseline brain volume and (3) a nonsignificant greater hippocampal volume decrease than placebo-treated patients.

These findings were contrary to predictions. However, increased titer-related losses in brain volume among antibody responders were not reflected in worsening cognitive performance among these individuals. ${ }^{19,23}$ These investigators concluded that there was a dissociation between brain volume loss and cognitive function in AN1792/QS-21 antibody responders; that is, whereas brain volume decreased in antibody responders to $A \beta$ antigens, cognitive functioning actually improved. The opinion of Fox et $a l^{23}$ is that the mechanisms underlying this dissociation remain unclear, but they include the possibility that the observed brain volume changes were because of (1) amyloid removal both within brain parenchyma and within the CSF, ${ }^{24-26}$ together with (2) possible cerebral fluid shifts. Fox et al. ${ }^{27}$ have presented further analyses of their own data set, with evidence for cortical and medial temporal lobe gray matter volume losses after treatment with AN1792/ QS-21, but relative preservation of brain stem and cerebellum. The authors also noted that although serial MRI used to track progression of atrophy in AD represents an objective and powerful measure, a number of (1) technical hurdles and (2) questions regarding interpretation of findings present themselves when MRI is used in multicenter clinical trials.

The neuropsychological findings presented by Gilman et al. ${ }^{19}$ and Fox et al. ${ }^{23}$ are significant, not least because they suggest that in-depth, quantitative neuropsychological testing may be more sensitive to the effect of immunotherapy than other forms of functional assessment (with the latter exemplified, in these studies, by the ADAS-Cog, DAD, Clinical Dementia Rating scale, MMSE or CGIC). Detailed, standardized neuropsychometric measures may therefore represent more accurate measures of cognitive and functional change subsequent to AD immunotherapy, when compared with the nonstandardized questionnaires and screening instruments that are often used as the outcome variables in many clinical trials. These questionnaires and screening instruments typically manifest lesser objectivity, test-retest reliability, and construct and predictive validity than quantitative, standardized neuropsychological measures. This is of especial concern when it is considered that such nonstandardized questionnaires and screening instruments (yielding qualitative rather than quantitative findings) are also used by many bedside clinicians as a central element of their diagnostic decision-making.
The phase IIa immunotherapy trial of AN1792 (QS-21) was interrupted because of meningoencephalitis in a proportion of immunized patients. As previously noted, both central activation of cytotoxic $\mathrm{T}$ cells and autoimmune reactions have been proposed as potential mechanisms underlying toxicity of AN1792. Neuropathological investigations were undertaken in two of the AN1792 patients affected by meningoencephalitis reported by Nicoll et $a .^{24}$ and by Ferrer et $a .^{25}$ These investigations revealed the presence of an unusual form of meningoencephalitis and leukoencephalopathy in these patients. Although amyloid plaques were sparse or absent throughout areas of the neocortex in these two patients (suggestive of favorable clearance of $A \beta$ ), other hallmarks of the $\mathrm{AD}$ brain, including cerebral amyloid angiopathy and neurofibrillary tangles, were identified in the CNS in these individuals. In addition, $\mathrm{T}$ lymphocytes and macrophages were present in the white matter of the brain in these two patients. ${ }^{24,25}$

The case report from Nicoll et al. ${ }^{24}$ was of a patient treated with AN1792 in whom a high A $\beta$ load was found in widespread brain regions. Specifically, the cerebral cortex of this patient contained concentrations of cerebral amyloid angiopathy similar to levels found in advanced AD. In this patient, the $A \beta$ load in the medial frontal gyrus was as high as in advanced $\mathrm{AD}$, and $\mathrm{A} \beta$ plaques were numerous in the basal ganglia and cerebellum. Commenting on these findings, Robinson et al. $^{28}$ argue that it is difficult to envisage how AN1792-induced serum antibodies to $A \beta$ could have resulted in the selective clearance of plaques from some areas of neocortex yet spared the very high densities of $A \beta$ that were observed in most other brain regions in this patient (including extracortical $A \beta$ plaques).

In addition, in a single case study, Masliah et al. ${ }^{26}$ reported a detailed investigation of a patient with $\mathrm{AD}$ without encephalitis who was immunized with AN1792. No amyloid plaques were noted in the frontal cortex in this case. Abundant $A \beta$-immunoreactive macrophages were noted, but neurofibrillary tangles and amyloid angiopathy were also present, indicating ongoing pathology. In this patient, the white matter appeared normal, and minimal lymphocytic infiltration of the leptomeninges was observed. However, related considerations to those raised by Robinson et $a .^{28}$ are again relevant when seeking to interpret these findings: according to Masliah et al., ${ }^{26}$ AN1792-induced serum antibodies apparently resulted in the selective clearance of some forms of amyloid in this patient whereas other signs of ongoing $\mathrm{AD}$ pathology continued to be present. It is important to note that these reports ${ }^{24-26}$ present data obtained from only a small number of patients treated with AN1792. Moreover, a major shortcoming of the above studies is that (using the methodology that was employed in these investigations) it was not possible to determine the level of brain amyloid in these particular patients before the onset of treatment. 
Future studies employing amyloid imaging (for example, PIB-PET) before and after treatment for AD should provide more definitive insights into the role of immunotherapy in the clearance of cerebral $A \beta$.

As already noted, the main AN1792 study was discontinued due to issues of safety and tolerability after the patients with encephalitis were identified. However, further empirical investigations have subsequently been conducted, to examine longer-term sequelae of active immunotherapy. A year later, a follow-up study was conducted by Hock and colleagues to assess ongoing safety of the AN1792 vaccine. $^{29,30}$ Of 30 patients studied, it was found that in patients who developed antibodies against A $\beta$ deposits $(n=20), \mathrm{AD}$ appeared to have stabilized clinically and functionally, rather than progressing (as might have been expected) over the 12 months of the followup. Furthermore, this apparent stabilization was noted in two (out of the three) treated patients who had previously developed encephalitis. It has therefore been suggested that patients with high levels of $\mathrm{A} \beta$ antibodies following treatment with the AN1792 vaccine were essentially protected from disease progression over the 1-year follow-up of the study. Nevertheless, this conclusion must be qualified by the consideration that the follow-up study ${ }^{29,30}$ included only $10 \%$ of the patients who had taken part in the original vaccination trial. Moreover, these supplementary findings do not indicate the level of putative brain tissue protection over the 1-year follow-up, as might have been revealed through neuroimaging investigations. If observed, the latter could represent central evidence for a true disease-modifying therapy.

Hock et $a .^{30}$ further considered the cognitive performance of $\mathrm{AD}$ patients in the phase IIa trials who had acquired high antibody titers against $A \beta$. They reported that over the 12-month follow-up period, a 'control' group (with no detectable A $\beta$ antibody titer; $N=9$ ) underwent a mean decline on the MMSE of 6.3 points. By contrast, Hock et al. report that the high $\mathrm{A} \beta$ antibody titer group $(N=19)$ declined by only 1.4 points on the MMSE over this same time period. However, Robinson et al. ${ }^{28}$ have noted that all of the high $A \beta$ antibody titer patients cited by Hock et al. ${ }^{30}$ were concurrently treated with acetylcholinesterase inhibitors. (This is an important consideration, because acetylcholinesterase inhibitors have been shown to slow the rate of cognitive deterioration in AD-as evaluated on the MMSE-to a mean decline of 1.5 points after 1 year of treatment. ${ }^{31,32}$ ) It should further be noted that the MMSE was one of the tests that failed to show significant antibody-related differences in test performance in the original AN1792 study findings reported by Gilman et al. ${ }^{19}$

Robinson et $a l^{28}$ also note that the rate of decline over a 12-month period of the control group reported by Hock et al. ${ }^{30}$ was exceptionally high (6.3 points on the MMSE), and that the results of the cognitive findings should therefore be interpreted cautiously. Furthermore, at the time of publication of their findings, the results presented by Hock et al. were still 'blinded'. Therefore, comparisons were made between (1) patients who either had or did not have an appreciable antibody titer, rather than between (2) patients who had been vaccinated with AN1792 or treated with placebo. The latter may be regarded as perhaps representing a fairer and more crucial comparison when evaluating the potential efficacy of AN1792 therapy; indeed, this may be considered a more general criticism of published studies in this field.

We have earlier suggested that detailed, standardized neuropsychological measures likely represent more sensitive measures of cognitive and functional change subsequent to AD immunotherapy, compared with subjective questionnaires and screening instruments. In this context, we have already noted that Hock et al. ${ }^{30}$ reported reduced decline in antibody responders with respect to scores on the MMSE (a relatively crude, brief screening instrument). By contrast, as Robinson et $a .^{28}$ identify, in the full AN1792 trial patients received a more thorough neuropsychological and functional assessment, which included the ADAS-Cog and the CGIC. Robinson et al. ${ }^{28}$ contend that the latter tests are more widely used as primary end points in pharmaceutical trials than the MMSE, and that it is therefore noteworthy that the patients with high $\mathrm{A} \beta$ antibody titers investigated by Hock et al. ${ }^{30}$ showed no significant differences on these neuropsychological and functional tasks compared with controls.

Seeking to characterize further the human antibody response to the pre-aggregated synthetic A $\beta 1-42$ preparation AN1792 vaccine (QS-21), Hock et al. ${ }^{29}$ collected sera from patients with $\mathrm{AD}$ who received a primary injection of pre-aggregated $A \beta 1-42$ followed by one booster injection of the same agent. Antibodies that were identified in responsive patients' immune sera recognized $A \beta$ plaques, diffuse $A \beta$ deposits and vascular $A \beta$ in brain blood vessels. The antibodies did not cross-react with native full-length amyloid precursor protein (that is, the parent molecule to $A \beta$ ) or its physiological derivatives, including soluble A $\beta 1-42$. On the basis of these findings, Hock et al. ${ }^{29}$ argued, optimistically, that vaccination of AD patients with $A \beta 1-42$ induced the production of antibodies that have a high degree of selectivity for the histopathogenic feature of AD. However, whether these $A \beta$ antibodies halt or decelerate cognitive decline in $\mathrm{AD}$ remains an open question, as we have already considered.

Hock et al. ${ }^{29}$ examined in further detail five patients from the AN1792 trials who had not displayed any adverse encephalitic symptoms. Two of these patients were reported to have detectable antibodies to $A \beta$ in their CSF. In both cases, the CSF titer was very low (1:50), even though these patients had high serum titers ( $>1: 1000$ and $>1: 10000$ ) of antibodies to $A \beta$. By contrast, one patient with meningoencephalitis had a high $A \beta$ antibody titer in their CSF ( $>1: 1000$ ), which was-in fact-higher than the titer found in 
that patient's serum $(1: 800)$. The presence of $A \beta$ antibodies in the CSF of meningoencephalitis patients is supported by Orgogozo et al., ${ }^{21}$ who found that six out of eight patients tested had detectable levels of $A \beta$ antibody in their CSF, whereas baseline measures for all of these patients had been negative for these antibodies.

Hock et al. ${ }^{30}$ further measured the concentrations of $\mathrm{A} \beta$ peptide in the serum and CSF of $20 \mathrm{AD}$ patients in the phase IIa trials of AN1792 who had acquired high antibody titers against $A \beta$. These researchers found that $A \beta 1-40$ and $A \beta 1-42$ peptide levels in the CSF and serum remained stable across the 12-month period of their investigation. Furthermore, the levels of $A \beta 1-40$ and $A \beta 1-42$ peptide levels in the CSF and serum did not differ from those in 10 'control' patients who had either not been vaccinated with $\mathrm{A} \beta$, or had been vaccinated but had not acquired an elevated titer of antibodies in their serum. ${ }^{30}$ It has been suggested by Robinson et $a .^{28}$ that these data indicate that AN1792 successfully elevated the titer of serum antibodies to $\mathrm{A} \beta$, but that-neverthelessthese antibodies did not successfully stimulate the removal of $A \beta$ plaques from the brains of these patients. Robinson et $a .^{28}$ further argue that this outcome is perhaps not unexpected in light of the insolubility of $A \beta$ plaques in humans. However, these findings contrast with the interpretation of their MRI findings by Fox et al., ${ }^{23}$ discussed earlier, who suggested that the observed brain volume reductions that they observed in AN1792 antibody responders were because of amyloid removal both within brain parenchyma and within the CSF, together with possible cerebral fluid shifts.

It is to be hoped that the findings reported to date by Gilman et al. ${ }^{19}$ and Fox et al. ${ }^{23}$ will be extended in further, long-term studies of AN1792 and similar agents, especially given the somewhat unexpected MRI findings, the incidence of meningoencephalitis and the observation-at one of the follow-up study sites-of reduced decline on the MMSE in the A $\beta$ antibody titer group of $\mathrm{AD}$ patients with respect to performance on the $\mathrm{MMSE}^{30}$ (notwithstanding that this was a psychometric instrument that was apparently insensitive to AN1792 intervention in the original comparison $^{19}$ ). However, analyses of data need to be standardized across studies with respect to key comparisons. This should help to clarify the impact of findings and reduce heterogeneity of neurocognitive outcomes across studies. Specifically, comparisons should be standardized in a clear, hypothesis-driven manner across centers participating in immunotherapy trials. As already noted, we favor a direct 'head to head' comparison between vaccinated patients and patients treated with placebo in evaluating the potential efficacy of immunotherapeutic agents, rather the application of a relatively arbitrary criterion for the definition of 'antibody responders' (such as that applied in the AN1792 trials).

Until recently, published data from the AN1792 were limited to short-term follow-up of patients who participated in the trial. This situation was rectified in June 2007 at the Alzheimer's Association International Conference on Prevention of Dementia in Washington, DC. Dr Michael Grundman, Senior Director of Clinical Development in the AD Program at Élan Pharmaceuticals, presented 4.5-year follow-up data on participants from the first major AN1792 AD immunotherapy trial (Élan-Wyeth). 159 patient/caregiver pairs took part in the AN1792 follow-up (30 placebo patients and 129 patients who had received AN1792). Of the 129 patients previously treated with AN1792, 25 were now classed as ongoing antibody responders. Compared to the placebo group, Grundman reported that the antibody responders showed significant favorable results in terms of maintained activities of daily living and leisure pursuits, reduced dependency on caregivers, and preserved memory and thinking skills. Moreover, after the first year of the follow-up, Grundman argued that brain volume changes in antibody responders and placebo patients were similar. No further cases of encephalitis were reported by Grundman in the 4.5-year follow-up. With respect to these findings, the presence of anti-A $\beta$ antibodies in patients after halting antigen injection several years earlier indicated a sustained response from the immune system. One possible mechanism for this could be the enhanced clearance of $A \beta$ from the CNS after immunotherapy into the periphery, where it may trigger an ongoing immune response. Alternatively, over this long time period the ongoing immune response to $A \beta$ could be present more widely, so as to recognize endogenous levels of the protein and overcome physiological tolerance to the presence of $\mathrm{A} \beta$ in the $\mathrm{AD}$ brain.

More detailed long-term findings were reported in 2008 by Holmes et al., ${ }^{33}$ who offered an important insight into the long-term effects that AN1792 treatment exerts on the immune response, $A \beta$ load and clinical and cognitive outcomes. In this review, the authors conducted a follow-up study from June 2003 to September 2006 on patients who had entered the phase 1 arm of the original AN1792 trial. Survival curves were constructed for all 80 participants in the trial. Post-mortem analysis was available on nine participants, all of whom were from the active treatment arm of the original study (one patient was subsequently excluded as this person were found to have progressive supranuclear palsy upon neuropathological examination). The authors noted that the degree of plaque removal and brain $A \beta$ load was variable among the eight cases available. However, the mean $A \beta$ load was reduced in immunized cases compared to nonimmunized controls. There was no observable relationship between AN1792 dose and A $\beta$ plaque removal or load. However, the degree of plaque removal varied in proportion to the mean antibody response generated over the treatment study period. One of the eight patients who were reviewed died suddenly 4 months after the study began, with a last recorded MMSE score of 16. The seven remaining patients manifested severe, end-stage dementia at the 
time of death. Interestingly, two of these patients had evidence of presumed extensive plaque removal in the cortex. There were no significant relationships between (1) AN1792 dose or (2) mean antibody response and (3) probability of survival (as assessed by time to death and hazard ratio). Likewise, there were no differences between placebo and active treatment groups in long-term survival outcomes or time to severe dementia, and no difference between groups on the outcome measures was assessed in the trial protocol, namely ADAS-Cog, MMSE or DAD.

To summarize, studies of active immunization have produced some evidence that, notwithstanding the caveats adumbrated earlier, AN1792 treatment may benefit elements of cognitive functioning in some patients with probable $\mathrm{AD}^{19,30}$ in the short term. However, the serious adverse side effects of meningoencephalitis in a percentage of patients mean that treatment with AN1792 cannot be considered a safe or tolerable treatment for $\mathrm{AD}$ at the present time. Similarly, the persistence over the longer term of AD symptoms in AN1792-treated patients suggests that plaque removal per se may not be sufficient in mid-tolate stages of disease pathogenesis to preserve cognitive and functional status. Interest in active immunization has not diminished, however. For example, the use of $A \beta$ fragments $(A \beta 4-10)$ has been shown to be effective in inhibiting $A \beta$ aggregation and cytoxicity in an AD mouse model, without inducing an inflammatory response. ${ }^{15}$ Such approaches warrant further investigation. Moreover, although the use of refined antigens and other $A \beta$-related immunotherapeutic agents may have therapeutic potential, alternative immunization methods have also shown promising results in clinical trials. Specifically, a beneficial outcome has been demonstrated using a non-A $\beta$ immunotherapy: nasal vaccination has been attempted successfully using the agent glatiramer acetate, a compound that has been used to treat multiple sclerosis ${ }^{34}$ (applied in combination with a proteosome-based adjuvant). This agent has been shown to reduce $\mathrm{A} \beta$ plaques in an $\mathrm{AD}$ mouse model, without the inflammatory effects shown by $A \beta$ immunotherapies. ${ }^{35}$ Such interventions may want further investigation in future human trials.

Agadjanyan et al. ${ }^{36}$ argue that the adverse response to AN1792 culminating in meningoencephalitis, observed in some cases in the original AN1792 trials, ${ }^{19}$ may have been caused by a T-cell-mediated autoimmune response. These researchers have developed a prototype epitope vaccine that contains the immunodominant B-cell epitope of $A \beta$ in tandem with the synthetic universal Th cell pan HLA DR epitope (pan HLA DR-binding peptide). This agent lacks the T-cell epitope of $A \beta$, with the associated potential to avoid the adverse events that occurred in the first clinical trial of AN1792. Similar agents lacking T-cell epitopes were reported by Mandler and colleagues at the Alzheimer's Association International Conference in Alzheimer's Disease in Chicago in July 2008. Furthermore, Solomon ${ }^{37}$ has suggested that the inclusion of an EFRH sequence within the immunogen-adjuvant may prevent T-cell autoimmune activation. In future, it may also be possible for the T-cell epitope found within $A \beta$ to be engineered out of potential immunotherapeutic preparations (thereby avoiding the generation of cross-reactive $\mathrm{T}$ cells). But less optimistically, it should be noted that in the original vaccination studies there was apparently no systematic association between the anti-A $\beta$ titers and the T-cell response.

\section{Passive immunization}

A complementary type of clinical trial is currently seeking to incorporate successful elements of active immunization trials, but without the adverse side effects. In this type of trial, participants receive injections of already prepared antibodies (IVIg), instead of an A $\beta$ preparation. IVIg is a purified human natural immunoglobulin preparation (with distinctive immune-modulating properties) that contains a collection of many antibodies, including natural human antibodies against $A \beta$. The passive immunization approach has been motivated by findings in nonhumans that IVIg promotes clearance of $A \beta$ from the brain, ${ }^{38}$ and that anti-A $\beta$ antibodies bind $A \beta$ and prevent oligomer formation. ${ }^{39}$ In addition, it has been observed that $\mathrm{AD}$ patients have considerably fewer antibodies to $A \beta$ in their blood than non-AD controls. ${ }^{40,41}$

A pilot study conducted by Dodel et $a .^{42}$ reported that cognitive functioning was stabilized in five patients with $\mathrm{AD}$ who were treated with IVIg for 6 months. Relkin et $a .^{43}$ infused anti-A $\beta$ antibodies into $\mathrm{AD}$ patients at various frequencies (from once per week, to once per month). The findings of this phase I study indicated that the majority of treated patients showed better cognitive functioning (as evaluated by the MMSE) after 6 months of IVIg administration, suggesting that this treatment may slow, halt or even reverse progression of the disease. Relkin et al. ${ }^{43}$ further commented that cognitive decline was arrested in all of their treated patients who had undergone psychometric testing. Specifically, the authors reported that over the 6 months of the study their patients improved on average 3 points on the MMSE. (By contrast, Relkin et al. ${ }^{43}$ argue that AD patients would typically fall 1.5 points on the MMSE over the same time period if they were left untreated.)

Relkin et $a .^{43}$ reported that all of their patients tolerated IVIg well. They further noted that anti-A $\beta$ antibodies increased significantly in plasma in a dose-dependent manner after each IVIg infusion, and that these levels rose incrementally with successive treatments, consistent with an IVIg half-life of approximately 10 days. Follow-up in the study also included measurements of blood A $\beta$ levels. There was a significant increase in plasma levels of $A \beta$ after IVIg infusion, interpreted as indicating that the $A \beta$ peptide was being mobilized by the IVIg treatment (specifi- 
cally, the authors inferred that the infused anti-A $\beta$ antibody had bound to endogenous $A \beta$, and was clearing $A \beta$ from the CSF to the plasma).

In addition to reporting changes in the blood, these researchers ${ }^{43}$ reported that anti-A $\beta$ antibodies were detected (and the level of $A \beta$ decreased) in the CSF of the patients subsequent to IVIg treatment. These findings were interpreted as a possible sign that the administered IVIg antibodies had crossed the bloodbrain barrier, and were inducing a decrease in $A \beta$ concentration in the brain. (It is thought that elements of IVIg bind to $A \beta$, making it more difficult for this protein to aggregate in the CNS.) Indeed, it has been shown that IgG-A $\beta$ complexes have higher permeability at the blood-brain barrier than IgG alone, ${ }^{44}$ suggesting that facilitation by a receptor-mediated transport mechanism indeed occurs. Recently, an IgM antibody has been produced that can be transported out across the blood-brain barrier and reverse cognitive deficits in mice. ${ }^{45}$

Additional results using passive immunotherapy in human trials have subsequently been reported by Relkin and colleagues. ${ }^{46-49}$ However, because these studies involved a relatively small number of $\mathrm{AD}$ patients, at this stage treatment with IVIg is promising but requires validation. In addition to replicating the findings obtained to date using passive immunotherapy in a larger group of patients, in future researchers will also need to determine definitively whether IVIg infusion impacts on the concentration of $A \beta$ in the brain. Furthermore, neurocognitive functioning after passive immunotherapy has, to date, been evaluated by means of a relatively crude and cognitively nonspecific instrument (the MMSE) ${ }^{43}$ supplemented by anecdotal reports (such as whether patients are able to narrate stories and socialize). Future studies should also use more specific, objective markers of neurocognitive capacity, notably measures of delayed episodic memory (which has been shown to be the most sensitive cognitive marker of decline in the early stages of AD). In addition, in the follow-up open-label study reported by Relkin and colleagues at the International Conference on Alzheimer's Disease and Related Disorders in Madrid in July 2006, ${ }^{46}$ it was noted that resumption of treatment with IVIg resulted in decreased plasma $A \beta$. The authors express uncertainty regarding the mechanisms underlying this subsequent effect, but suggest that this phenomenon bears further study in a larger set of AD patients treated chronically and continuously with IVIg.

Although both passive and active immunization therapies may act through similar mechanisms, a benefit of passive immunization is that it uses an existing plasma product (IVIg) with current Food and Drug Administration approval that (1) has been utilized with good clinical tolerability for several decades, (2) has been used effectively in the treatment of hundreds of thousands of patients suffering from a range of immune-deficiency and auto-immune conditions and (3) has an established safety record (thereby reducing the time and risks associated with developing and testing novel treatments for AD). In addition, passive immunization is attractive because of the greater therapeutic control over anti-A $\beta$ titers, and the potential to rapidly terminate the treatment if adverse events occur. On the basis of previous results, larger scale phase II and phase III clinical trials are currently underway or planned to determine optimal dosing of IVIg in AD.

However, some research conducted in laboratory mice has suggested that IVIg immunotherapy might lead to increased congophilic angiopathy and vascular leakage. ${ }^{50}$ Furthermore, even if IVIg were to be approved by regulatory bodies as an effective therapy in $\mathrm{AD}$, there is currently not enough IVIg available to treat all AD patients. The drug is produced in limited quantities, and prescription of this agent costs around US\$3000-7000 per month. A further potential criticism of IVIg is that it represents a less sophisticated and less targeted approach compared to (1) the more specific active immunization methodology, or (2) the administration of humanized antibodies directed specifically against $A \beta$ epitopes.

However, Relkin and colleagues have suggested that there are multiple types of antibodies in IVIg against the amyloid molecule. Consistent with his notion, it has been argued by Istrin et $a .^{38}$ that IVIg interferes by more than one mechanism in clearing $A \beta$ from the brains of Alzheimer's patients. It may be possible in future to filter out the specific antiamyloid antibodies from the pool of antibodies within IVIg, creating a potentially more focused and powerful treatment. Studies are currently underway to determine which available immunoglobulin products contain the highest possible levels of anti-A $\beta 1-42$ antibodies. ${ }^{51}$ Furthermore, it has been suggested that IVIg may attach to both the soluble and insoluble forms of $A \beta$ (driving $A \beta$ out to the periphery, where it may be cleared). Passive immunization may target both the N-terminal and central region epitopes of $A \beta$, whereas it may be possible to fine-tune active immunization, such that it targets only the former. (Indeed this has been attempted with active agents such as CAD106, developed by the Novartis Institutes for Biomedical Research.) Heppner et al. ${ }^{20}$ argue that 'more fine-tuning in guiding the immune response is needed to circumvent detrimental side effects' in $\mathrm{AD}$, and have suggested that focused N-terminal treatments may be less likely to elicit detrimental outcomes involving cytotoxic T-cell responses. However, it is noteworthy that, to date, adverse treatment side effects have been identified in studies using active immunization treatment rather than subsequent to passive immunization. With respect to its proposed mechanism of action, nonhuman research has indicated that passive immunization may work effectively to reduce amyloid toxicity. ${ }^{52}$ Furthermore, passive immunization may operate in conjunction with endogenous mechanisms, specifically related to a shift in microglial phenotype. ${ }^{53}$ Indeed, the effects of IVIg on the clearance of $A \beta$ by microglia cells have been demonstrated in cellular models. ${ }^{38}$ 
On the basis of other findings, ${ }^{54,55}$ it has been suggested that human monoclonal antibodies (resembling physiologically normal nonpathogenic and possibly protective antibodies in healthy adults) might be attractive candidates for immune therapy in $\mathrm{AD}$, perhaps used in combination with IVIg. In animal models, passive immunization used in conjunction with monoclonal antibodies has demonstrated significant improvements in spatial learning and memory in mice. ${ }^{56}$ Further, it is likely that epitope-specific monoclonal anti-A $\beta$ antibodies would have advantages over IVIg antibodies in enhancing effects on $A \beta$ load. There may be some further advantages to the use of modified fragments of monoclonal antibodies (i.e., excluding the $\mathrm{Fc}$ portion, but retaining the $\mathrm{F}(\mathrm{ab})_{2}$ component) that have been shown to bind $A \beta$ plaques. ${ }^{57}$ These modified fragments have advantages over whole IgG molecules, which include (1) increased permeability at the blood-brain barrier (thus potentially increasing clearance of $A \beta$ out from the CNS), and (2) minimizing the inflammatory response and associated hemorrhage. Further, recent evidence suggests that monoclonal antibodies can be developed to specifically target neurotoxic $A \beta$ oligomers and not $A \beta$ monomers, ${ }^{58}$ with significant potential for future methods of passive immunotherapy. Taken together, it is anticipated that these features of monoclonal antibody therapies are likely to enhance the clearance/degradation of $\mathrm{A} \beta$ from the $\mathrm{AD}$ brain. In addition, other therapeutic antibodies such as the monoclonal antibody m266 are currently being investigated as possible agents for passive immunotherapy in $\mathrm{AD},{ }^{59}$ whereas catalytic immunoglobulins (such as $\operatorname{IgV}_{\mathrm{L}}$ ) are thought to offer particular promise because multiple molecules of $A \beta$ can be inactivated by each catalytic immunoglobulin molecule.

The latest research reports indicate that passive immunization may offer potential over a more extended time period. Specifically, in July 2008 at the Alzheimer's Association International Conference in Alzheimer's Disease in Chicago, Relkin and colleagues reported that in a phase II trial of 24 patients with mild-to-moderate AD treatment with IVIg over 9 months (without a washout period) produced further statistically significant improvements on both cognitive and global clinical measures. Similar findings were reported at the same meeting by Huang et al. A phase III clinical trial of IVIg in AD is currently under way. Relkin and colleagues further reported meaningful changes in CSF proteins with known $A \beta$-binding properties after treatment with IVIg, and proposed that conformational human antibodies to misfolded proteins may represent an important component of the therapeutic effects of IVIg in AD patients. Relkin and colleagues further proposed at the 2008 International Conference in Alzheimer's Disease that human antibodies within IVIg alter the assembly of amyloidogenic peptides into soluble oligomers (possibly underlying the clinical efficacy of IVIg in the three clinical trials conducted to date).

\section{Conclusions}

As stated by Morgan, ${ }^{60}$ the attitudes toward immunotherapy for the treatment of $\mathrm{AD}$ have followed something of a roller coaster ride. Initial interest in the potential of this treatment was substantially tempered by the adverse outcomes in the initial clinical trials. ${ }^{19}$ However, as discussed in this article, active and passive immunotherapeutic approaches are currently being pursued to try to address some of the negative outcomes that have been previously associated with immunization treatments in AD.

At the present time, it would appear that immunization does offer some potential as a technique for the treatment (and even prevention) of AD. However, future studies are needed to tackle key outstanding issues. For example, effective vaccines need to minimize T-cell responses and provide greater benefits for cognition than those agents that have been tested so far. Robinson et $a .^{28}$ have further suggested that the most important lesson to be learned from the AN1792 clinical trials that have been conducted to date is that new strategies for treating AD should not be tested on humans until they have been tested on non-murine animal species, such as primates. These authors further argue that extant findings using AN1792 illustrate how little we truly understand about the interrelationships between $A \beta$ antibodies, $\mathrm{AD}$ and meningoencephalitis, and that-although this situation persists-it would be imprudent to undertake further human trials using active immunization. These authors further argue that even passive immunization has risks for humans, and it should be performed with utmost caution only after extensive testing on animals. ${ }^{61}$ In essence, Robinson et al. ${ }^{28}$ argue that the pathophysiology of AD is characterized by much more than accumulation of $A \beta$ in the brain, and it is therefore unlikely that the prevention and/or clearance of $A \beta$ deposition by immunotherapy will offer an effective treatment for the disease.

Overall, although acknowledging that there are several important caveats, we are a little more sanguine than Robinson et $a .^{28}$ regarding the future potential of immunotherapy in $\mathrm{AD}$. We believe that our incremental understanding of the pathophysiological basis of $\mathrm{AD}$ will lead to safer forms of immunotherapy. Further, we regard current antiamyloid methodologies as hopeful but not yet conclusive. Specifically, subsequent neuropathological examinations from the immunotherapy trials conducted to date have indicated that there were decreased deposits of $A \beta$ in individuals who were immunized with AN1792.. ${ }^{24,25}$ Furthermore, some positive cognitive and functional outcomes were additionally reported in both the original and follow-up active immunotherapy studies, and after treatment with passive immunization. ${ }^{19,23,30,43}$ Moreover, it has been suggested that the placebo- 
treated AD patients in the AN1792 studies did not decline functionally (as would normally be expected) over the course of the trial, such that it was thereby more difficult to demonstrate a significant, beneficial effect of AN1792 treatment. In addition, it may have been problematic to recruit unequivocally diagnosed $\mathrm{AD}$ cases for the original AN1792 clinical trials. In this context, those positive outcomes of the clinical trials that were obtained may offer some proof of concept regarding the potential value of immunotherapy. Future laboratory studies may also reveal the source of the pathogenesis underlying the meningoencephalitis that arose in some patients who participated in the AN1792 active immunization trials.

As outlined by Nitsch at the Alzheimer's Association International Conference in Alzheimer's Disease in Chicago in July 2008, other immunotherapy studies in humans and animals have indicated improved neurite and synapse morphology, reduced astrocytosis and restored neural functions (including long-term potentiation and behavior). There is also the potential in future of stimulating innate immunity in $\mathrm{AD}$ patients by Toll-like receptors. However, future caution is warranted, with outstanding challenges for immunotherapy including autoimmune disease, brain inflammation, microhemorhage, increased amyloid angiopathy, remaining neurofibrillary tangles, reductions in brain volume, and problems with blood-brain barrier passage of antibodies and with the precise molecular definition of the therapeutic target. Specifically, it has been suggested that oligomeric and fibrillar forms of $A \beta$ may manifest different susceptibilities to antibody treatment. ${ }^{62}$ Further, some nonhuman studies have indicated that anti-A $\beta$ antibodies may preferentially clear small $A \beta$ aggregates associated with cognitive dysfunction, but this effect may not be necessarily associated with lowering the overall $A \beta$ load in the brain. ${ }^{20}$ Moreover, it is also possible that in different subgroups of AD patients the ratio of excess $A \beta$ deposition vs increased neuro inflammation varies, and, therefore, vaccine therapy may be effective for some subgroups of $\mathrm{AD}$ patients but not for other subgroups. This consideration, in turn, impacts upon the issue of patient sample size in future vaccine trials, and better definition of patient subgroups. Related to this last point, in addition to immunotherapy, other forms of treatment may be appropriate in $\mathrm{AD}$, and 'one size will not necessarily fit all' with respect to the treatment of different patients. Therefore, immunotherapy may not be a universal treatment. In future, larger trials that systematically investigate patient subgroups may offer more definitive outcomes. Finally, immunotherapy might be most beneficial in concert with other therapies, for example, anti-inflammation drugs and/ or diabetes drugs aimed at reducing the inflammatory effects of $A \beta$.

There have been some suggestions that 'nano bodies' (generated by other species), which represent a simpler form of antibody than humans produce, could be used to treat diseases such as AD by passive immunotherapy. ${ }^{63,64}$ It has been noted that nanobodies are much less complex than human antibodies, so it may be easier to derive smaller fragments that could then be used effectively in AD treatment. It appears that the small size of nanobodies may permit them to access brain tissue more effectively than would be the case for conventional therapies. Furthermore, the nanobody methodology may be able to combine multiple functions into the same molecule, whereas comparison of different nanobodies may yield insight into whether enhancing the capacity of distinct immunotherapeutics to cross the blood-brain barrier results in increased clinical efficacy in AD.

Another important consideration concerns the relationship between nonhuman research findings and potential clinical application in humans. In animal models, both soluble forms of $A \beta$ and fibrillar plaques are implicated in cognitive impairment. However, the characteristic pathophysiology identified in patients with $\mathrm{AD}$ is somewhat different and more diverse. Specifically, in addition to $A \beta$-related pathological changes, intraneuronal neurofibrillary lesions and significant neuronal and synaptic losses occur even in patients with the earliest symptoms of AD. In addition, as noted earlier, animal models do not mimic $\mathrm{AD}$ with respect to issues pertaining to amyloid clearance, as vaccination of nonhuman animals removes induced amyloid deposits whereas vaccination in humans targets naturally occurring amyloid. It remains an open question whether the vaccination approach will be able to tackle effectively the range of pathological processes evident in the brain of human $\mathrm{AD}$ patients, and repair already damaged neural systems. Perhaps the vaccine will halt progression of neurodegeneration in human $\mathrm{AD}$ patients, and subsequently brain plasticity mechanisms will allow some recovery of function.

Other therapeutic $A \beta$ vaccination strategies that are currently being pursued include immunoconjugates and monoclonal antibodies, although amplicon vectors derived from agents such as the herpes simplex virus ${ }^{65}$ and direct genetic therapies have also been suggested as suitable methods for the treatment of $\mathrm{AD}$. Moreover, it is possible that active and passive immunization approaches may be synergistic, and thereby could be used effectively in tandem. Finally, it may be the case that therapeutic anti-amyloid trials may be most revealing in early onset, familial $\mathrm{AD}$ carriers who already have amyloid pathology but no symptoms (with PIB-PET amyloid imaging undertaken before and after treatment).

With respect to $A \beta$, although the vaccination studies conducted to date have demonstrated a role for immune and inflammatory cells in clearing this protein, it still remains to be determined whether the central mechanism involves promoting degradation of $A \beta$, increased clearance of $A \beta$ from the brain/CSF, or a combination of both of these mechanisms. McGeer and $\mathrm{McGeer}^{66}$ have argued that vaccination with $\mathrm{A} \beta$ 
in AD patients will increase complement activation through formation of antigen-antibody complexes. In transgenic mice, these authors suggest that this process will enhance phagocytosis. But in AD patients, where complement is already overactivated, and where the senile plaques are relatively insoluble, McGeer and McGeer have argued that this stimulation should increase production of the membrane attack complex, thereby actually increasing the autodestruction of neurons in AD. Furthermore, in the postmortem tissue available to date (and from active immunization attempts in transgenic animal models), immunization was associated with a lower $A \beta$ plaque load in some brain regions; however, the persistence of amyloid that has been observed in cerebral blood vessels in patients ${ }^{24-26}$ is puzzling and may relate to plaque removal and presumed re-solublization of $A \beta$.

There are other outstanding clinical and ethical questions. Specifically, the issue of determining necessary and sufficient signs for treatment is a key issue for ongoing clinical trials, given the potential toxic side effects of some of the immunotherapy agents that are currently being tested. So, even if a vaccine is effective in patients, will it be safe? Or will autoimmune issues always plague this approach, given that one is seeking to direct the body's immune system against an endogenous human protein? And what is a tolerable level of safety for such treatment regimes? For instance, the release of recent results from the phase II trial of Élan-Wyeth's passive vaccine, bapineuzumab, in July 2008 at the Alzheimer's Association International Conference in Alzheimer's Disease in Chicago indicated that some participants (the majority of whom were APOE 44 carriers) receiving active treatment experienced vasogenic edema. Should it be at the discretion of an individual who is manifesting early stage symptoms of $\mathrm{AD}$ (and who will likely progress to profound dementia over the next several years) to choose to take the risk of potentially serious side effects concomitant with immunotherapy? (Most of the clinical trial participants who presented with encephalitis after the original AN1792 study recovered, but one third of these patients currently remain chronically impaired.) And how should one define and apply the concept of informed consent to treatment in such a context (an especially germane issue in a condition such as $\mathrm{AD}$, where cognitive functioning, judgment and the capacity to give consent become compromised during the course of the disease)? If immunotherapy is shown to be both effective and safe (within tolerable limits), when should a physician choose to treat, given that current clinical methods for identifying individuals at high risk of developing AD are imperfect? ${ }^{67}$ Further, in light of the data from the bapineuzumab phase II trial, should clinical best practice be stratified on the basis of APOE 44 possession?

To conclude, future clinical studies using immunotherapy are needed to tackle key outstanding issues. These include (1) the need to demonstrate efficacy of
A $\beta$ vaccination in humans as well as in animal models (especially with respect to the issues of TAPIR, and regarding potentially toxic side effects of immunotherapy in humans), (2) the use of appropriate control comparisons and (3) fine-tuning in safely guiding the immune response. The vaccination methodology does appear to offer substantial current promise for clearing brain amyloid in $\mathrm{AD}$ patients-but is this sufficient for it to become, potentially, the first choice among future amyloidreducing treatment options? Moreover, the issue of determining the critical internationally agreed signs for clinical efficacy remains an additional issue for future immunization trials. New studies are seeking to use safer vaccines that do not result in toxicity and neuroinflammation. But it remains an open question whether the immunization approach will help to repair already damaged neural systems in $\mathrm{AD}$.

A vaccine that is safely able to clear amyloid plaques in the brains of AD patients would offer a valuable avenue in the search for a solution to the disease: not only would it offer us potentially more effective clinical therapies, but it would also enable us to determine whether the accumulation of $A \beta$ in the brain in $\mathrm{AD}$ is a key causal feature of the disease, or a functionally less significant consequence. These are both key goals in the search to find an effective treatment (and possible cure) for AD. If future clinical trials continue successfully to completion, they are therefore likely to offer both the first, direct therapeutic evaluations of the amyloid hypothesis in AD patients, and potentially the first clinically effective treatment of the disease.

\section{Acknowledgments}

GV was generously supported by a grant from Warren Milner (Milner English College-Perth, Western Australia) and Helen Sewell. KAB was supported through a grant from Wesfarmers Limited, Australia. JKF and RNM were supported by the WA Neurotrauma Research Program. RNM was supported by a program grant from the National Institute of Health. All authors were supported by the McCusker Foundation for Alzheimer's Disease Research, the WA Centre of Excellence for Alzheimer's Disease Research and Care, and the National Health and Medical Research Council of Australia. We are grateful to the referees of this paper for their generous and constructive feedback.

\section{References}

1 Lleo A, Greenberg SM, Growdon JH. Current pharmacotherapy for Alzheimer's disease. Annu Rev Med 2006; 57: 513-533.

2 Hardy J, Selkoe DJ. The amyloid hypothesis of Alzheimer's disease: progress and problems on the road to therapeutics. Science 2002; 297: 353-356.

3 Kemppainen NM, Aalto S, Wilson IA, Nagren K, Helin S, Bruck A et al. Voxel-based analysis of PET amyloid ligand [C-11]PIB uptake in Alzheimer disease. Neurology 2006; 67: 575-1580. 
4 Shaw LM, Korecka M, Clark CM, Lee VMY, Trojanowski JQ. Biomarkers of neurodegeneration for diagnosis and monitoring therapeutics. Nat Rev Drug Discov 2007; 6: 295-303.

5 Selkoe DJ. Toward a comprehensive theory for Alzheimer's disease-hypothesis: Alzheimer's disease is caused by the cerebral accumulation and cytotoxicity of amyloid beta-protein. Ann NY Acad Sci 2000; 924: 17-25.

6 Fiala M, Lin J, Ringman J, Kermani-Arab V, Tsao G, Patel A et al. Ineffective phagocytosis of amyloid-beta by macrophages of Alzheimer's disease patients. J Alzheimers Dis 2005; 7: 221-232.

7 Patterson C, Feightner J, Garcia A, MacKnight C. General risk factors for dementia: a systematic evidence review. Alzheimer Demen 2007; 3: 341-347.

8 Aksari P, Stoppe G. Risk factors of Alzheimer's disease-a review. Fortschr Neurol Psychiatr 1996; 64: 425-432.

9 Martins IJ, Hone E, Foster JK, Sunram-Lea SI, Gnjec A, Fuller SJ et al. Apolipoprotein E, cholesterol metabolism, diabetes, and the convergence of risk factors for Alzheimer's disease and cardiovascular disease. Mol Psychiatry 2006; 11: 721-736.

10 Stozicka Z, Zilka N, Novak M. Risk and protective factors for sporadic Alzheimer's disease. Acta Virol 2007; 51: 205-222.

11 Schenk D, Barbour R, Dunn W, Gordon G, Grajeda H, Guido T et al. Immunization with amyloid-beta attenuates Alzheimer diseaselike pathology in the PDAPP mouse. Nature 1999; 400: 173-177.

12 Janus C, Pearson J, McLaurin J, Mathews PM, Jiang Y, Schmidt SD et al. Abeta peptide immunization reduces behavioural impairment and plaques in a model of Alzheimer's disease. Nature 2000; 408: $979-982$.

13 Morgan D, Diamond DM, Gottschall PE, Ugen KE, Dickey C, Hardy J et al. Abeta peptide vaccination prevents memory loss in an animal model of Alzheimer's disease. Nature 2000; 408: 982-985.

14 DeMattos RB, Bales KR, Cummins DJ, Dodart JC, Paul SM, Holtzman DM. Peripheral anti-Abeta antibody alters CNS and plasma Abeta clearance and decreases brain Abeta burden in a mouse model of Alzheimer's disease. Proc Natl Acad Sci USA 2001; 98: 8850-8855.

15 McLaurin J, Cecal R, Kierstead ME, Tian X, Phinney AL, Manea M et al. Therapeutically effective antibodies against amyloid-beta peptide target amyloid-beta residues 4-10 and inhibit cytotoxicity and fibrillogenesis. Nat Med 2002; 8: 1263-1269.

16 Brendza RP, Bacskai BJ, Cirrito JR, Simmons KA, Skoch JM, Klunk WE et al. Anti-Abeta antibody treatment promotes the rapid recovery of amyloid-associated neuritic dystrophy in PDAPP transgenic mice. J Clin Invest 2005; 115: 428-433.

17 Becker M, Lavie V, Solomon B. Stimulation of endogenous neurogenesis by anti-EFRH immunization in transgenic mice model of Alzheimer's disease. Proc Natl Acad Sci USA 2007; 104: 1691-1696.

18 Foster JK. Hippocampus, recognition, and recall: a new twist on some old data? Behav Brain Sci 1999; 22: 449.

19 Gilman S, Koller M, Black RS, Jenkins L, Griffith SG, Fox NC et al. Clinical effects of Abeta immunization (AN1792) in patients with AD in an interrupted trial. Neurology 2005; 64: 1553-1562.

20 Heppner FL, Gandy S, McLaurin J. Current concepts and future prospects for Alzheimer disease vaccines. Alzheimer Dis Assoc Disord 2004; 18: 38-43.

21 Orgogozo JM, Gilman S, Dartigues JF, Laurent B, Puel M, Kirby LC et al. Subacute meningoencephalitis in a subset of patients with AD after Abeta 42 immunization. Neurology 2003; 61: 46-54.

22 Morris RG, Kopelman MD. The memory deficits in Alzheimer-type dementia—a review. Q J Exp Psychol A 1986; 38: 575-602.

23 Fox NC, Black RS, Gilman S, Rossor MN, Griffith SG, Jenkins L et al. Effects of Abeta immunization (AN1792) on MRI measures of cerebral volume in Alzheimer disease. Neurology 2005; 64: 1563-1572.

24 Nicoll JAR, Wilkinson D, Holmes C, Steart P, Markham H, Weller RO. Neuropathology of human Alzheimer disease after immunization with amyloid-beta peptide: a case report. Nat Med 2003; 9: 448-452.

25 Ferrer I, Rovira MB, Guerra MLS, Rey MJ, Costa-Jussa F. Neuropathology and pathogenesis of encephalitis following amyloid-beta immunization in Alzheimer's disease. Brain Pathol 2004; 14: 11-20.

26 Masliah E, Hansen L, Adame A, Crews L, Bard F, Lee C et al. Abeta vaccination effects on plaque pathology in the absence of encephalitis in Alzheimer disease. Neurology 2005; 64: 129-131.
27 Fox N. Detecting change in Alzheimer's disease brains. Alzheimers Demen 2006; 2: S46.

28 Robinson SR, Bishop GA, Lee HG, Munch G. Lessons from the AN 1792 Alzheimer vaccine: lest we forget. Neurobiol Aging 2004; 25: 609-615.

29 Hock C, Konietzko U, Papassotiropoulos A, Wollmer A, Streffer J, von Rotz RC et al. Generation of antibodies specific for betaamyloid by vaccination of patients with Alzheimer disease. Nat Med 2002; 8: 1270-1275.

30 Hock C, Konietzko U, Streffer JR, Tracy J, Signorell A, MullerTillmanns B et al. Antibodies against beta-amyloid slow cognitive decline in Alzheimer's disease. Neuron 2003; 38: 547-554.

31 Klatte ET, Scharre DW, Nagaraja HN, Davis RA, Beversdorf DQ. Combination therapy of donepezil and vitamin $\mathrm{E}$ in Alzheimer disease. Alzheimer Dis Assoc Disord 2003; 17: 113-116.

32 Winblad B, Engedal K, Soininen H, Verhey F, Waldemar G, Wimo A et al. A 1-year, randomized, placebo-controlled study of donepezil in patients with mild to moderate AD. Neurology 2001; 57: 489-495.

33 Holmes C, Boche D, Wilkinson D, Yadegarfar G, Hopkins V, Bayer A et al. Long-term effects of Abeta42 immunisation in Alzheimer's disease: follow-up of a randomised, placebo-controlled phase I trial. Lancet 2008; 372: 180-182.

34 Johnson KP, Brooks BR, Cohen JA, Ford CC, Goldstein J, Lisak RP et al. Copolymer-1 reduces relapse rate and improves disability in relapsing-remitting multiple sclerosis-results of a phase-III multicenter, double-blind, placebo-controlled trial. Neurology 1995; 45: 1268-1276.

35 Frenkel D, Maron R, Burt DS, Weiner HL. Nasal vaccination with a proteosome-based adjuvant and glatiramer acetate clears betaamyloid in a mouse model of Alzheimer disease. I Clin Invest 2005; 115: 2423-2433.

36 Agadjanyan MG, Ghochikyan A, Petrushina I, Vasilevko V, Movsesyan N, Mkrtichyan M et al. Prototype Alzheimer's disease vaccine using the immunodominant $B$ cell epitope from betaamyloid and promiscuous T cell epitope pan HLA DR-binding peptide. J Immunol 2005; 174: 1580-1586.

37 Solomon B. Alzheimer's disease immunotherapy: from in vitro amyloid immunomodulation to in vivo vaccination. In: Perry G, Avila J, Kinoshita J, Smith MA (eds). Alzheimer's Disease: A Century of Scientific and Clinical Research. IOS Press: The Netherlands, 2006, pp 433-438.

38 Istrin G, Bosis E, Solomon B. Intravenous immunoglobulin enhances the clearance of fibrillar amyloid-beta peptide. $J$ Neurosci Res 2006; 84: 434-443.

39 Ma QL, Lim GP, Harris-White ME, Yang F, Ambegaokar SS, Ubeda OJ et al. Antibodies against beta-amyloid reduce Abeta oligomers, glycogen synthase kinase-3beta activation and tau phosphorylation in vivo and in vitro. J Neurosci Res 2006; 83: 374-384.

40 Bacskai BJ, Kajdasz ST, Christie RH, Carter C, Games D, Seubert P et al. Imaging of amyloid- $\beta$ deposits in brains of living mice permits direct observation of clearance of plaques with immunotherapy. Nat Med 2001; 7: 369-372.

41 Bard F, Cannon C, Barbour R, Burke RL, Games D, Grajeda H et al. Peripherally administered antibodies against amyloid beta-peptide enter the central nervous system and reduce pathology in a mouse model of Alzheimer disease. Nat Med 2000; 6: 916-919.

42 Dodel RC, Du Y, Depboylu C, Hampel H, Frolich L, Haag A et al. Intravenous immunoglobulins containing antibodies against betaamyloid for the treatment of Alzheimer's disease. I Neurol Neurosurg Psychiatry 2004; 75: 1472-1474.

43 Relkin N, Szabo P, Adamiak B, Monthe C, Burgut FT, Du YS et al. Intravenous immunoglobulin (IVIg) treatment causes dose-dependent alterations in beta-amyloid (Abeta) levels and anti-beta antibody titres in plasma and cerebrospinal fluid (CSF) of Alzheimer's disease (AD) patients. Neurology 2005; 64(Suppl 1): A144-A145.

44 Poduslo JF, Curran GL. Amyloid beta peptide as a vaccine for Alzheimer's disease involves receptor-mediated transport at the blood-brain barrier. Neuroreport 2001; 12: 3197-3200.

45 Banks WA, Farr SA, Morley JE, Wolf KM, Geylis V, Steinitz M et al. Anti-amyloid beta protein antibody passage across the bloodbrain barrier in the SAMP8 mouse model of Alzheimer's disease: an age-related selective uptake with reversal of learning impairment. Exp Neurol 2007; 206: 248-256. 
46 Relkin NR, Younkin L, Younkin S, Monthe C, Adamiak B, Szabo P et al. Decreased plasma beta amyloid levels in Alzheimer patients treated chronically with Intravenous immunoglobulin (IVIg). Alzheimers Demen 2006; 2: S590.

47 Relkin NR. Intravenous immunoglobulin (IVIg) therapy. Alzheimers Demen 2006; 2: S51.

48 Szabo P, Esteban M, Monthé C, White L, Lent R, Relkin N et al. Increasing the efficacy of immunotherapy for Alzheimer's disease (AD) with intravenous immunoglobulin (IVIg). Alzheimers Demen 2006; 2: S361.

49 Adamiak B, Monthe C, Bender H, Szabo P, Schiff R, Weksler ME et al. Intravenous immunoglobulin (IVIg) maintains cognition over 18 months in patients with Alzheimer's disease (AD). Alzheimers Demen 2006; 2: S62-S63.

50 Pfeifer M, Boncristiano S, Bondolfi L, Stalder A, Deller T, Staufenbiel M et al. Cerebral hemorrhage after passive anti-Abeta immunotherapy. Science 2002; 298: 1379.

51 Safavi A. Comparison of several human immunoglobulin products for anti-Abeta 1-42 titer. Alzheimers Demen 2006; 2: S591.

52 Mohajeri MH, Saini K, Schultz JG, Wollmer MA, Hock C, Nitsch RM. Passive immunization against beta-amyloid peptide protects central nervous system (CNS) neurons from increased vulnerability associated with an Alzheimer's disease-causing mutation. J Biol Chem 2002; 277: 33012-33017.

53 Morgan D. Modulation of microglial activation state following passive immunization in amyloid depositing transgenic mice. Neurochem Int 2006; 49: 190-194.

54 Geylis V, Kourilov V, Meiner Z, Nennesmo I, Bogdanovic N, Steinitz M. Human monoclonal antibodies against amyloid-beta from healthy adults. Neurobiol Aging 2005; 26: 597-606.

55 Tamura Y, Hamajima K, Matsui K, Yanoma S, Narita M, Tajima N et al. The $\mathrm{F}(\mathrm{ab})(2)$ fragment of an A beta-specific monoclonal antibody reduces A beta deposits in the brain. Neurobiol Dis 2005; 20: $541-549$.

56 Lee EB, Leng LZ, Zhang B, Kwong L, Trojanowski JQ, Abel T et al. Targeting amyloid-beta peptide (Abeta) oligomers by passive immunization with a conformation-selective monoclonal antibody improves learning and memory in Abeta precursor protein (APP) transgenic mice. J Biol Chem 2006; 281: 4292-4299.
57 Poduslo JF, Ramakrishnan M, Holasek SS, Ramirez-Alvarado M, Kandimalla KK, Gilles EJ et al. In vivo targeting of antibody fragments to the nervous system for Alzheimer's disease immunotherapy and molecular imaging of amyloid plaques. J Neurochem 2007; 102: 420-433.

58 Lambert MP, Velasco PT, Chang L, Viola KL, Fernandez S, Lacor PN et al. Monoclonal antibodies that target pathological assemblies of A-beta. J Neurochem 2007; 100: 23-35.

59 DeMattos RB, Racke MM, Bryan MT, Siemers ER. Passive immunotherapy with M266 results in altered CSF A $\beta$. Alzheimers Demen 2006; 2: S589.

60 Morgan D. Immunotherapy for Alzheimer's disease. In: Perry G, Avila J, Kinoshita J, Smith MA (eds). Alzheimer's Disease: A Century of Scientific and Clinical Research. IOS Press: The Netherlands, 2006, pp 425-432.

61 Lee EB, Leng LZ, Lee VMY, Trojanowski JQ. Meningoencephalitis associated with passive immunization of a transgenic murine model of Alzheimer's amyloidosis. FEBS Letters 2005; 579: 2564-2568.

62 Agadjanyan M, Mamikonyan G, Necula M, Mkrtichyan M, Ghochikyan A, Petrushina I et al. Different susceptibilities of oligomeric and fibrillar forms of $\beta$-amyloid to antibodies specific to N-terminus of this peptide. Alzheimers Demen 2006; 2: S613S614.

63 Merchiers P, van Dooren T, Van der Auwera I, Abulrob A, Lauwereys M, Roland B et al. Nanobodies targeting amyloid beta as potential therapeutics for Alzheimer's disease. Alzheimers Demen 2006; 2: S589.

64 Van Dooren T, Merchiers P, Borghgraef P, Dewachter I, Van der Auwera I, Wera S et al. Target specificity of nanobodies directed against amyloid peptides. Alzheimers Demen 2006; 2: S598-S599.

65 Bowers WJ, Mastrangelo MA, Stanley HA, Casey AE, Milo LJ, Federoff HJ. HSV amplicon-mediated Abeta vaccination in Tg2576 mice: differential antigen-specific immune responses. Neurobiol Aging 2005; 26: 393-407.

$66 \mathrm{McGeer}$ PL, McGeer E. Is there a future for vaccination as a treatment for Alzheimer's disease? Neurobiol Aging 2003; 24: 391-395.

67 Foster JK, Sohrabi H, Verdile G, Martins RN. Research criteria for the diagnosis of Alzheimer's disease: genetic risk factors, blood biomarkers and olfactory dysfunction. Int Psychogeriatr 2008; 20 853-855. 